\title{
ON COMMUTATIVE $V^{*}$-ALGEBRAS II
}

\author{
by P. G. SPAIN \\ (Received 18 September, 1970)
}

1. Introduction. We show that the commutative $V^{*}$-algebras with relatively weakly compact unit spheres are those that are representable by means of hermitian spectral measures. This provides a more unified approach to the results of [15], and allows us to generalise some of them.

Let $X$ be a complex Banach space with dual space $X^{\prime}$ and semi-inner-product [,] compatible with the norm. Let $\left\langle x, x^{\prime}\right\rangle$ be the value of the functional $x^{\prime}$ in $X^{\prime}$ at the point $x$ in $X$. When $Y$ is a subset of $X$, we write $Y^{w}$ for the weak closure of $Y$. Let $\mathscr{L}(X)$ be the algebra of bounded linear operators on $X$. When $\mathscr{T}$ is a subset of $\mathscr{L}(X)$, we write $\mathscr{T}^{w}$ for the closure of $\mathscr{T}$ in the weak (operator) topology and $\mathscr{T}^{s}$ for the closure of $\mathscr{T}$ in the strong (operator) topology. We write $\mathscr{T}_{k}$ for $\{T \in \mathscr{T}:\|T\| \leqq k\}$.

For $x$ in $X$, we define the point state $\omega_{x}: \mathscr{L}(X) \rightarrow \mathrm{C}: T \mapsto[T x, x] . \quad T$ is said to be hermitian if $W(T)=\left\{\omega_{x}(T):\|x\|=1\right\}$, the numerical range of $T$, is a set of real numbers. (These topics are discussed in [10].)

Let $\mathscr{A}$ be a closed subalgebra of $\mathscr{L}(X)$ and let $\mathscr{H}$ be the set of hermitian operators in $\mathscr{A}$. $\mathscr{A}$ is called a $V^{*}$-algebra if $I \in \mathscr{A}$ and $\mathscr{A}=\mathscr{H}+i \mathscr{H}$. Then $\mathscr{A}$ is a $V^{*}$-algebra if and only if $I \in \mathscr{A}$ and $\mathscr{A}$ is a $C^{*}$-algebra under the operator norm and the (Vidav) involution ${ }^{*}: R+i J \mapsto$ $R-i J(R, J \in \mathscr{H})[11]$.

We refer the reader to [15] for the definitions of (possibly unbounded) normal, selfconjugate and strongly self-conjugate operators. A bounded operator is normal if and only if it is contained in a commutative $V^{*}$-algebra.

Let $\Lambda$ be a compact Hausdorff space and let $C(\Lambda)$ be the space of continuous complex functions on $\Lambda$, with the supremum norm. Let $S(\Lambda)\left(S_{0}(\Lambda)\right)$ be the family of Borel (Baire) sets of $\Lambda$; let $B(\Lambda)\left(B_{0}(\Lambda)\right)$ be the space of bounded Borel (Baire) measurable functions on $\Lambda$, with the supremum norm.

We refer to [5] for the definition and properties of spectral measures.

Throughout this paper, $\mathscr{A}$ will be a commutative $V^{*}$-algebra on $X$ with maximal ideal space $\Lambda$ and inverse Gelfand map $\psi: C(\Lambda) \rightarrow \mathscr{A}$. $\mathscr{H}$ will be the set of hermitian operators in $\mathscr{A}$.

\section{Commutative $V^{*}$-algebras with weakly compact unit spheres.}

Lemma 1. If $T \in \mathscr{H}$, then $\left(I+T^{2}\right)^{-1} \in \mathscr{H}_{1}$ and $2 T\left(I+T^{2}\right)^{-1} \in \mathscr{H}_{1}$. Conversely, if $S \in \mathscr{H}_{1}$, there is $a T$ in $\mathscr{H}$ such that $S=2 T\left(I+T^{2}\right)^{-1}$.

Proof. This lemma holds in any $C^{*}$-algebra with identity. (See Lemma 6 of $[14$, p. 24].)

LEMMA 2. There is a unique regular spectral measure $F(\cdot)$ of class $(S(\Lambda), X)$ with values in $\mathscr{L}\left(X^{\prime}\right)$ such that

$$
\left\langle\psi(f) x, x^{\prime}\right\rangle=\int_{\Lambda} f(\lambda)\left\langle x, F(d \lambda) x^{\prime}\right\rangle \quad\left(x \in X, x^{\prime} \in X^{\prime}, f \in C(\Lambda)\right)
$$


Proof. For $x$ in $X$ and $x^{\prime}$ in $X^{\prime}$, the map $\psi_{x, x^{\prime}}: C(\Lambda) \rightarrow \mathrm{C}: f \mapsto\left\langle\psi(f) x, x^{\prime}\right\rangle$ is a functional on $C(\Lambda)$ bounded by $\|x\|\left\|x^{\prime}\right\|$. By the Riesz representation theorem, there is a unique regular Borel measure $\mu\left(\cdot ; x, x^{\prime}\right)$ on $\Lambda$ such that $\left\|\mu\left(\cdot ; x, x^{\prime}\right)\right\| \leqq\|x\|\left\|x^{\prime}\right\|$ and

$$
\text { - }\left\langle\psi(f) x, x^{\prime}\right\rangle=\int_{\Lambda} f(\lambda) \mu\left(d \lambda ; x, x^{\prime}\right) \quad\left(x \in X, x^{\prime} \in X^{\prime}, f \in C(\Lambda)\right) .
$$

For $\tau$ in $S(\Lambda)$ and $x^{\prime}$ in $X^{\prime}$, the map $x \mapsto \mu\left(\tau ; x, x^{\prime}\right)$ is a bounded functional on $X$. Therefore there is an $F_{x^{\prime}}(\tau)$ in $X^{\prime}$ such that $\mu\left(\tau ; x, x^{\prime}\right)=\left\langle x, F_{x^{\prime}}(\tau)\right\rangle$. Clearly $\left\|F_{x^{\prime}}(\tau)\right\| \leqq\left\|x^{\prime}\right\|$. By linearity and the uniqueness of $\mu\left(\cdot ; x, x^{\prime}\right)$, there is an operator $F(\tau)$ in $\mathscr{L}\left(X^{\prime}\right)$ such that $F_{x^{\prime}}(\tau)=F(\tau) x^{\prime}$. Also $\|F(\tau)\| \leqq 1$. Since each $\mu\left(\cdot ; x, x^{\prime}\right)$ is a regular measure and $\psi$ is an algebra isomorphism, it is routine to check that $F(\cdot)$ is a regular spectral measure of class $(S(\Lambda), X)$. Then $\|F(\tau)\|=1(\tau \in S(\Lambda))$, since each $F(\tau)$ is a projection.

Lemma 3. $\|S x\|=\left\|S^{*} x\right\|(x \in X, S \in \mathscr{A})$.

Proof. Let $S=\psi(f)$; then $S^{*}=\psi(f)$. We define $g$ on $\Lambda$ by $g(\lambda)=f(\lambda) / f(\lambda)$ if $f(\lambda) \neq 0$, $g(\lambda)=0$ if $f(\lambda)=0$. Then $g \in B(\Lambda)$ and $\|g\|=1$. We define $U$ in $\mathscr{L}\left(X^{\prime}\right)$ by $U=\int_{\Lambda} g(\lambda) F(d \lambda)$. For $x$ in $X$ and $x^{\prime}$ in $X^{\prime}$, we have

$$
\left|\left\langle x, U x^{\prime}\right\rangle\right|=\left|\int_{\Lambda} g(\lambda) \mu\left(d \lambda ; x, x^{\prime}\right)\right| \leqq\|g\|\left\|\mu\left(\cdot ; x, x^{\prime}\right)\right\| \leqq\|x\|\left\|x^{\prime}\right\| .
$$

Therefore $\|U\| \leqq 1$. Also, $\left(S^{*}\right)^{\prime}=S^{\prime} U$. Hence

$$
\left\|S^{*} x\right\|=\sup _{\left\|x^{\prime}\right\| \leqq 1}\left|\left\langle S^{*} x, x^{\prime}\right\rangle\right|=\sup _{\left\|x^{\prime}\right\| \leqq 1}\left|\left\langle x, S^{\prime} U x^{\prime}\right\rangle\right|=\sup _{\left\|x^{\prime}\right\| \leqq 1}\left|\left\langle S x, U x^{\prime}\right\rangle\right| \leqq\|S x\| .
$$

By symmetry, $\left\|S^{*} x\right\|=\|S x\|$.

Lemmas 2 and 3 are similar to Theorem 2.5(ii) and Lemma 2.7 of [12]. The first part of the next theorem is the same as Theorem 2.8 of [12].

THEOREM 1. $\mathscr{A}^{w}$ is a commutative $V^{*}$-algebra and $\left(\mathscr{A}^{w}\right)_{1}=\left(\mathscr{A}_{1}\right)^{w}$.

Proof. If $S \in \mathscr{A}^{w}$, there is a net $\left\{S_{s}=R_{s}+i J_{s}: s \in \sigma\right\}$ in $\mathscr{A}$ with strong limit $S$. Lemma 3 shows that $\left\{S_{s}^{*}\right\}$ converges strongly. Hence $\left\{R_{s}: s \in \sigma\right\}$ and $\left\{J_{s}: s \in \sigma\right\}$ converge strongly to $R$ and $J$ in $\mathscr{H}^{w}$ and $S=R+i J$. Hence $\mathscr{A}^{w}=\mathscr{H}^{w}+i \mathscr{H}^{w}$. Therefore $\mathscr{A}^{w}$ is a commutative $V^{*}$-algebra and $\mathscr{H}^{w}$ is the set of hermitian operators in $\mathscr{A}^{w}$.

Let $S \in\left(\mathscr{H}^{w}\right)_{1}$ and let $T$ in $\mathscr{H}^{w}$ be such that $S=2 T\left(I+T^{2}\right)^{-1}$. Let $\left\{T_{s}: s \in \sigma\right\}$ be a net in $\mathscr{H}$ converging strongly to $T$; put $S_{s}=2 T_{s}\left(I+T_{s}^{2}\right)^{-1}$. Then, as in [14, p. 25, Theorem 2] or $[6$, p. 47],

$$
S_{s}-S=2\left(I+T_{s}^{2}\right)^{-1}\left(T_{s}-T\right)\left(I+T^{2}\right)^{-1}+\frac{1}{2} S_{s}\left(T-T_{s}\right) S
$$

Therefore $S$ is the strong limit of $\left\{S_{s}\right\}$ in $\mathscr{H}_{1}$; so $\left(\mathscr{H}^{w}\right)_{1} \subset\left(\mathscr{H}_{1}\right)^{w}$.

By the Russo and Dye theorem [11, p. 538], $\left(\mathscr{A}^{w}\right)_{1} \subset\left(\mathscr{A}_{1}\right)^{w}$. Hence $\left(\mathscr{A}^{w}\right)_{1}=\left(\mathscr{A}_{1}\right)^{w}$.

Definition. We say that $\mathscr{A}$ is representable by a spectral measure if there is a regular hermitian spectral measure $E(\cdot)$ of class $\left(S(\Lambda), X^{\prime}\right)$ with values in $\mathscr{L}(X)$ such that $\psi(f)=$ $\int_{\Lambda} f(\lambda) E(d \lambda)(f \in C(\Lambda))$. Such a spectral measure is unique. 
THEOREM 2. $\mathscr{A}$ is representable by a spectral measure if and only if $\mathscr{A}_{1}$ is relatively weakly compact. If this is so, then $\mathscr{A}^{w}$ is a commutative $W^{*}$-algebra and any faithful representation of $\mathscr{A}^{w}$ as a von Neumann algebra is weakly and strongly bicontinuous on bounded spheres.

Proof. Let $\mathscr{A}$ be represented by the spectral measure $E(\cdot)$. For each $x$ in $X$ the map $\psi_{x}: C(\Lambda) \rightarrow X: f \mapsto \psi(f) x$ is weakly compact [1, Theorem 3.2]; hence $\mathscr{A}_{1} x$ is relatively weakly compact in $X$. The argument suggested in [7, p. 511, Exercise 2] shows that $\mathscr{A}_{1}$ is relatively weakly compact in $\mathscr{L}(X)$.

Let $\mathscr{A}_{1}$ be relatively weakly compact. Theorem 3 of [16] shows that any von Neumann representation $\phi: \mathscr{A}^{w} \rightarrow \mathscr{B} \subset \mathscr{L}(H)$ is weakly bicontinuous on bounded spheres; also, $\left(\mathscr{A}_{1}\right)^{w}=\left(\mathscr{A}^{w}\right)_{1}$. By [7, X.2.1] the map $\phi \psi: C(\Lambda) \rightarrow \phi \mathscr{A}$ has the form $f \mapsto \int_{\Lambda} f(\lambda) E^{h}(d \lambda)$, where $E^{h}(\cdot)$ is a unique regular hermitian spectral measure of class $(S(\Lambda), H)$ with values in $\mathscr{L}(H)$. Also $E^{h}(\tau) \in \mathscr{B}(\tau \in S(\Lambda))$. We define $E(\cdot)$ by $E(\cdot)=\phi^{-1} E^{h}(\cdot)$. Then $E(\cdot)$ is a regular hermitian spectral measure of class $\left(S(\Lambda), X^{\prime}\right)$, since $\phi$ is weakly bicontinuous on bounded spheres; and $\psi(f)=\int_{\Lambda} f(\lambda) E(d \lambda)(f \in C(\Lambda))$ because $\phi$ is an isometry.

Suppose that $\mathscr{A}_{1}$ is relatively weakly compact. We have still to prove that any von Neumann representation $\phi$ of $\mathscr{A}^{w}$ is strongly bicontinuous on bounded spheres.

Let $\left\{T_{s}: s \in \sigma\right\}$ be a bounded net in $\mathscr{A}^{w}$ and let $\lim _{\sigma} T_{s}=0$ in the strong topology. Then $\lim _{\sigma} \omega_{x}\left(T_{s}^{*} T_{s}\right)=0(x \in X)$. We next show that the converse holds.

Let $\tilde{\Lambda}$ be the maximal ideal space of $\mathscr{A}^{w}$, let $\tilde{E}(\cdot)$,be its representing spectral measure and $\tilde{\psi}$ the inverse Gelfand map defined by $\tilde{\psi}: C(\bar{\Lambda}) \rightarrow \mathscr{A}^{w}: f \mapsto \int_{\bar{\lambda}} f(\lambda) \tilde{E}(d \lambda)$. Let $f_{s}=\psi^{-1} T_{s}$ $(s \in \sigma)$. Since $\left\{T_{s}\right\}$ is a bounded net and the weak topology on a bounded sphere is the weak topology induced by the point states [16, Lemma 1], it follows that

$$
\lim _{\sigma}\left\langle T_{s}^{*} T_{s} x, x^{\prime}\right\rangle=\lim _{\sigma} \int_{\tilde{\lambda}}\left|f_{s}(\lambda)\right|^{2}\left\langle\tilde{E}(d \lambda) x, x^{\prime}\right\rangle=0 \quad\left(x \in X, x^{\prime} \in X^{\prime}\right) .
$$

Therefore $\lim _{\sigma} f_{s}=0$ in $\operatorname{var}\left(\left\langle E(\cdot) x, x^{\prime}\right\rangle\right)$-measure, and $\lim _{\sigma} \int_{X} f_{s}(\lambda)\left\langle E(d \lambda) x, x^{\prime}\right\rangle=0$. For fixed $x$ in $X$, the set $\left\{\left\langle\widetilde{E}(\cdot) x, x^{\prime}\right\rangle:\left\|x^{\prime}\right\| \leqq 1\right\}$ is a relatively weakly compact set of measures [7, IV.10.2]; hence, by [9, Théorème 2], $\lim _{\sigma} \int_{\pi} f_{s}(\lambda)\left\langle\widetilde{E}(d \lambda) x, x^{\prime}\right\rangle=0$ uniformly for $\left\|x^{\prime}\right\| \leqq 1$. Therefore $\lim _{\sigma} \int_{\AA} f_{s}(\lambda) \tilde{E}(d \lambda) x=0$; that is, $\lim _{\sigma} T_{s}=0$ in the strong topology.

Thus, if $\left\{T_{s}: s \in \sigma\right\}$ is a bounded net in $\mathscr{A}^{w}, \lim _{\sigma} T_{s}=0$ in the strong topology if and only if $\lim _{\sigma} T_{s}^{*} T_{s}=0$ in the weak topology. It follows that $\phi$ is strongly bicontinuous on bounded spheres.

REMARK 1. The hypotheses of the theorem hold if $E(\cdot)$ is a hermitian spectral measure of class $\left(S_{0}(\Lambda), X^{\prime}\right)$ and $\psi(f)=\int_{\Lambda} f(\lambda) E(d \lambda)(f \in C(\Lambda))$ [1, Theorem 3.2].

COROLlaRY 1. If $\mathscr{A}_{1}$ is relatively weakly compact and $\left\{R_{s}: s \in \sigma\right\}$ is a bounded monotone increasing net in $\mathscr{H}$, then $\underset{\sigma}{\mathrm{V}} R_{s}=\lim _{\sigma} R_{s}$ in the strong topology. 
Proof. We already know that $\bigvee_{\sigma} R$ exists and is the weak limit of $\left\{R_{s}\right\}$ [16, Lemma 2]. Let $\phi$ be a von Neumann representation of $\mathscr{A}^{w}$. Then $\underset{\sigma}{\bigvee_{\sigma}} \phi R_{s}=\lim _{\sigma} \phi R_{s}$ in the strong topology [6, Appendice II]; whence the result (cf. [4, Theorem 4.2] and [13, Lemma 3]).

COROLlary 2. Let $X$ be weakly (sequentially) complete. Then $\mathscr{A}$ is representable by a spectral measure.

Proof. For each $x$ in $X$, the map $\psi_{x}: C(\Lambda) \rightarrow X: f \mapsto \psi(f) x$ is weakly compact [7, VI. 7.6]. It follows as in the theorem that $\mathscr{A}_{1}$ is relatively weakly compact. (This corollary is Theorem 2.5(i) of [12].)

REMARK 2. Since any bounded Boolean algebra of projections can be made hermitian by a suitable equivalent renorming of $X[4, \S 3$, Remark 2], the theorem includes Corollary 2 to Theorem 3 of [8].

3. Applications of Theorem 2. The results of [15] are based on the use of Theorem 2.5(i) of [12] and Corollary 2 of Theorem 3 of [8]. These are both corollaries of Theorem 2 above.

Theorem 2 and the proof of Theorem 1 of [15] give our next result.

THEOREM 3. Let $T$ be a normal operator on $X$ and let $\mathscr{A}$ be the commutative $V^{*}$-algebra generated by $T$. Let $\mathscr{A}_{1}$ be relatively weakly compact. Then $\sigma(T)$ (the spectrum of $T$ ) is the maximal ideal space of $\mathscr{A}$. If $E(\cdot)$ is the representing spectral measure for $\mathscr{A}$, then $\lambda$ in $\sigma(T)$ is an eigenvalue of $T$ if and only if $E(\{\lambda\}) \neq 0$.

Theorem 2 and the proof of Theorem 2 of [15] give our next result.

THEOREM 4. Let $S$ be a strongly self-conjugate operator on $X$ and let its generated group of isometries $\{U(t, S): t \in \mathbf{R}\}$ be contained in a commutative $V^{*}$-algebra with relatively weakly compact unit sphere. Then there is a regular hermitian spectral measure $E(\cdot)$ of class $\left(S(\mathbf{R}), X^{\prime}\right)$ such that

$$
\begin{array}{rlr}
U(t, S) & =\lim _{n} \int_{-n}^{n} e^{i t \lambda} E(d \lambda) & (t \in \mathbf{R}), \\
S x & =\lim _{n} \int_{-n}^{n} \lambda E(d \lambda) x & (x \in \mathscr{D}(S)) .
\end{array}
$$

Theorem 5 of [15] may be further generalised.

THEOREM 5. Let $\mathscr{B}$ ' be a bounded Boolean algebra of projections on a Banach space $X$, and let the closed algebra generated by $\mathscr{B}$ ' have relatively weakly compact unit sphere. Then $\mathscr{B}^{\prime}$ has a $\sigma$-complete extension contained in $\left(\mathscr{B}^{\prime}\right)^{s}$.

Proof. By Remark 2, there is no loss of generality in assuming that each projection in $\mathscr{B}^{\prime}$ is hermitian. Let $\Lambda$ be the Stone space of $\mathscr{B}^{\prime}, K^{\prime}(\Lambda)$ the set of characteristic functions of open-and-closed subsets of $\Lambda$, and let $\psi^{\prime}: K^{\prime}() \rightarrow \mathscr{B}^{\prime}: \chi_{\tau} \mapsto B(\tau)$ be the representation isomorphism. We extend $\psi^{\prime}$ to an algebra isomorphism $\psi: K(\Lambda) \rightarrow \mathscr{B}: \sum c_{j} \chi_{\tau_{j}} \mapsto \sum c_{j} B\left(\tau_{j}\right)$, 
where $K(\Lambda)$ is the algebra generated by $K^{\prime}(), \mathscr{B}$ that generated by $\mathscr{B ^ { \prime }}$. Then $\psi$ is an isometry [3, Theorem 2.1].

Since $\Lambda$ is totally disconnected, $K(\Lambda)$ is norm dense in $C(\Lambda)$. We extend $\psi$ to an isometric isomorphism (also denoted by) $\psi: C(\Lambda) \rightarrow \overline{\mathscr{B}}$ (norm closure of $\mathscr{B}$ ). Then $\overline{\mathscr{B}}=K+i K$, where $K$ is the set of hermitian operators in $\mathscr{B}$. Thus $\mathscr{B}$ is a commutative $V^{*}$-algebra.

Let $E(\cdot)$ be the representing spectral measure for $\overline{\mathscr{B}}$. Let $\mathscr{B}=\left\{E(\tau): \tau \in S_{0}(\Lambda)\right\}$. Then $\mathscr{B}$ is a Boolean algebra of hermitian projections containing $\mathscr{B}^{\prime}$.

Let $\left\{E\left(\tau_{n}\right): n=1,2, \ldots\right\}$ be a sequence in $\mathscr{B}$ and let

$$
\tau=\bigcup_{1}^{\infty} \tau_{n}
$$

Put

Then

$$
\tau_{1}^{\prime}=\tau_{1}, \quad \tau_{n+1}^{\prime}=\tau_{n+1} \mid \bigcup_{1}^{n} \tau_{k} \quad(n=1,2, \ldots)
$$

$$
E(\tau)=\lim _{n} E\left(\bigcup_{1}^{n} \tau_{k}^{\prime}\right)=\lim _{n} \bigvee_{1}^{n} E\left(\tau_{k}^{\prime}\right)=\lim _{n} \bigvee_{1}^{n} E\left(\tau_{k}\right)
$$

where the limits exist in the strong topology (by the Banach-Orlicz-Pettis theorem). It is clear that $E(\tau) X=\operatorname{clm}\left\{E\left(\tau_{n}\right) X\right\}$.

The proof of the existence of $\Lambda E\left(\tau_{n}\right)$ and that $\left(\Lambda E\left(\tau_{n}\right)\right) X=\bigcap\left(E\left(\tau_{n}\right) X\right)$ is similar. Thus $\not{B}$ is $\sigma$-complete.

Since $\Lambda$ is totally disconnected, $S_{0}(\Lambda)$ is contained in the $\sigma$-algebra generated by the openand-closed sets. Hence $\mathscr{B} \subset\left(\mathscr{B}^{\prime}\right)^{s}$.

I would like to thank Professor E. Berkson for making his paper [4] available to me in advance of publication.

I would like to take this opportunity of thanking Dr H. R. Dowson who introduced me to the literature of $V^{*}$-algebras and who guided and encouraged me in this work.

\section{REFERENCES}

1. R. G. Bartle, N. Dunford and J. T. Schwartz, Weak compactness and vector measures, Canad. J. Math. 7 (1955), 289-305.

2. E. Berkson, A characterization of scalar type operators on reflexive Banach spaces, Pacific J. Math. 13 (1963), 365-373.

3. E. Berkson, Some characterizations of $C^{*}$-algebras, Illinois J. Math. 10 (1966), 1-8.

4. E. Berkson, Action of $W^{*}$-algebras in Banach spaces, Math. Ann. 189 (1970), 261-271.

5. E. Berkson and H. R. Dowson, Prespectral operators, Illinois J. Math. 13 (1969), 291-315.

6. J. Dixmier, Les algèbres d'opérateurs dans l'espace hilbertien (Paris, 1957).

7. N. Dunford and J. T. Schwartz, Linear operators (New York, 1958 and 1963).

8. D. A. Edwards and C. T. Ionescu Tulcea, Some remarks on commutative algebras of operators on Banach spaces, Trans. Amer. Math. Soc. 93 (1959), 541-551.

9. A. Grothendieck, Sur les applications linéaires faiblement compactes d'espaces du type $C(K)$, Canad. J. Math. 5 (1953), 129-173. 
10. G. Lumer, Semi-inner-product spaces, Trans. Amer. Math. Soc. 100 (1961), 29-43.

11. T. W. Palmer, Characterizations of $C^{*}$-algebras, Bull. Amer. Math. Soc. 74 (1968), 538-540.

12. T. W. Palmer, Unbounded normal operators on Banach spaces, Trans. Amer. Math. Soc. 133 (1968), 385-414.

13. T. V. Panchapagesan, Semigroups of scalar type operators in Banach spaces, Pacific J. Math. 30 (1969), 489-517.

14. J. R. Ringrose, Lecture notes on von Neumann algebras (Newcastle upon Tyne, 1967).

15. P. G. Spain, On commutative $V^{*}$-algebras, Proc. Edinburgh Math. Soc. (2) 17 (1970), 173-180. $62-64$.

16. P. G. Spain, $V^{*}$-algebras with weakly compact unit spheres, J.London Math. Soc. (2) 4 (1971),

UNIVERSITY OF GLASGOW

GLASGOW, G12 8QQ 